\title{
Kompetitive Allele Specific PCR (KASP): A Singleplex Genotyping Platform and Its Application
}

\author{
Uzma Majeed $^{1}$, Essam Darwish ${ }^{2}$, Shoaib Ur Rehman ${ }^{1} \&$ Xueyong Zhang ${ }^{1}$ \\ ${ }^{1}$ Key Laboratory of Crop Gene Resources and Germplasm Enhancement, Ministry of Agriculture/Institute of \\ Crop Science, Chinese Academy of Agricultural Sciences, Beijing, China \\ ${ }^{2}$ Department of Agricultural Botany, Plant Physiology Section, Faculty of Agriculture, Cairo University, Giza, \\ Egypt \\ Correspondence: Xueyong Zhang, Key Laboratory of Crop Gene Resources and Germplasm Enhancement, \\ Ministry of Agriculture/Institute of Crop Science, Chinese Academy of Agricultural Sciences, Beijing 100081, \\ China. Tel: 86-10-8210-6695. E-mail: zhangxueyong@caas.cn
}

Received: September 11, 2018

Accepted: October 25, 2018

Online Published: December 15, 2018

doi:10.5539/jas.v11n1p11

URL: https://doi.org/10.5539/jas.v11n1p11

The research is financed by grants from the National Key Research and Development Program of China (2016YFD0100302).

\begin{abstract}
Single nucleotide polymorphism (SNP) can be detected by mining sequence databases or by using different singleplex or multiplex SNP genotyping platforms. Development of high-throughput genotyping molecular markers can be instrumental towards maximizing genetic gain. In this review we provide an overview of Kompetitive Allele Specific PCR (KASP) genotyping platform requirements and its application that might be helpful in KASP marker development. This literature further illustrates the possibilities to design KASP primers. Several research institutes routinely using KASP platform, producing in excess of humungous data points yearly for breeding cultivars and as well as for medical and commercial purposes. KASP genotyping technology offers cost effectiveness and high throughput molecular marker development platform. Conventional molecular markers can be converted into more robust and high throughput KASP markers. More than 2000 published references clearly show the popularity of KASP technology among the researchers.
\end{abstract}

Keywords: Kompetitive Allele Specific PCR (KASP), singleplex assay, KASP primer designing, marker development

\section{Introduction}

Development of molecular markers that predict the trait of interest with highest level of accuracy offers efficient tracking of superior genetic variations through breeding process bypassing phenotypic evaluation requirements. Most of the molecular markers developed for marker assisted selection (MAS) are actually diagnostic markers which forecast phenotype with varying degree of accuracy. Hence, the availability of more robust and reliable molecular markers have become the matter of need than merely a research theme.

In recent times, single nucleotide polymorphisms (SNPs) have been widely used as markers in MAS. SNPs have largely replaced simple sequence repeats (SSRs) in crop species that have been comprehensively sequenced, such as maize and they are expected to substitute other kinds of molecular markers in most of the species in the forthcoming times given the large scale use of next generation sequencing technologies for genotyping (Semagn et al., 2013). SNPs are abundant in genomes and they are amenable for high throughput detection. Beside this, low cost assay, locus specificity, simple documentation, co-dominant inheritance and relatively low genotyping error rates (Schlotterer, 2004), SNPs have emerged as powerful tools for genomic selection. In past, simple sequence repeat (SSR) markers facilitated the development of high density maps for rice (Mccouch et al., 2002), barley (Varshney et al., 2007), wheat (Somers et al., 2004), maize (Smith et al., 1997), and other crops (Varshney et al., 2005). At present SNPs are considered more informative as compared to SSRs (Xu \& Crouch, 2008) because it is difficult to compare SSR data from different populations or platforms. Moreover, SSR motifs are 
finite in a genome and they are unevenly distributed. Gel-based SSR scoring is cost ineffective as genotyping is tedious and time consuming.

The continuous progress in high throughput genotyping technologies has resulted in various SNP genotyping platforms (Rasheed et al., 2017). In recent past, PCR based DNA fragment length polymorphism (DLFP) technology was widely used for the detection of variation in presence or absence of target sequences for restriction endonucleases. Cleaved amplified polymorphic sequences (CAPS) are analogy of restriction fragment length polymorphism (RFLP) but it needs an amplified product instead of original genomic DNA. Utility of CAPS is restricted only to detection of SNPs that creates or remove target site for the specific restriction enzyme. A slight modified form of CAPS is derived cleaved amplified polymorphic sequence (dCAPS) which are designed with 1 or 2 mismatches. Through these mismatches, primers create mutations into the target sequences during amplification and conjunction with the discovered SNP it results in formation of a unique restriction site. Both CAPS and dCAPS are gel-based genotyping methods. Scoring of genotype is laborious, time consuming and might cause error while scoring for large set of population (Figure 1).

To overcome these limitations to a considerable level, gel free high throughput single plex SNP genotyping platforms such as Kompetitive Allele Specific PCR (KASP ${ }^{\mathrm{TM}}$ ) is more suitable (Neelam et al., 2013). To get rid of post PCR handling requirements single tube assay such as KASP offer opportunities for high throughput genotyping by lowering the cost and time in assaying tens of hundreds of genotyping while reducing error rates (Kumpatla et al., 2012). KASP is homogenous, fluorescence labeled genotyping technology developed by KBioscience. KASP is based on allele specific oligo extension and fluorescence resonance energy transfer (FRET) for signal generation (Kumpatla et al., 2012). Fluorescent reporting system comprises of four single labeled oligonucleotides that hybridize to one another in free solution to form a fluorescent quenched pair which upon introduction of complementary sequences creates a measureable signal. The KASP genotyping can be carried out in 96, 384 and 1536 well PCR plates.

A

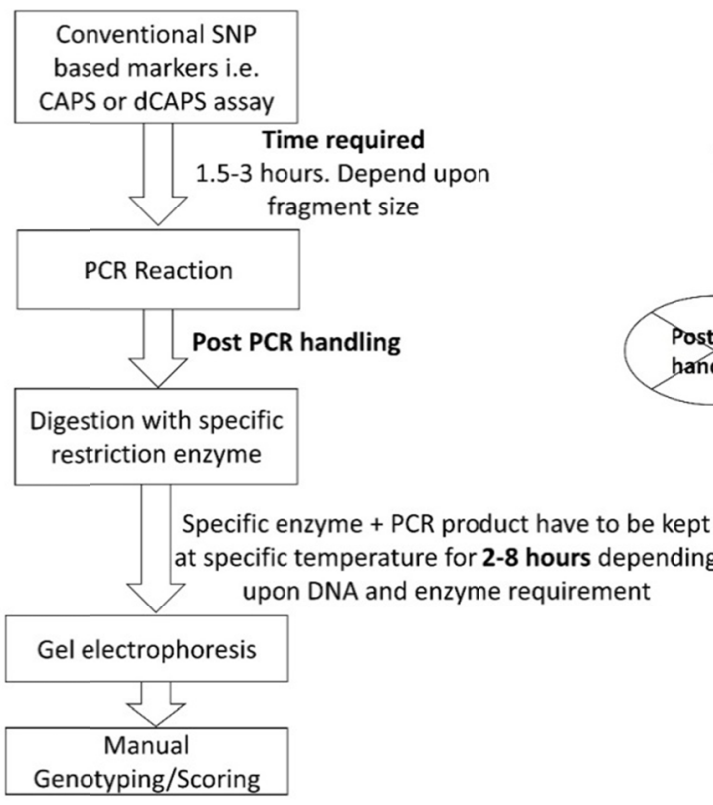

B

Latest SNP based markers i.e. KASP assay

Time required

1.5 hours.

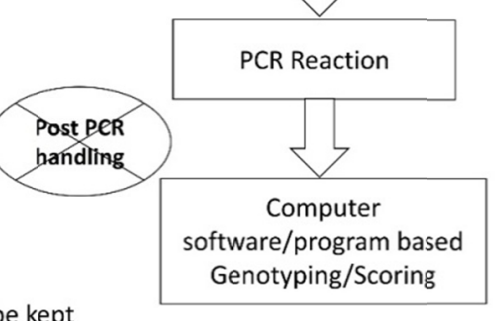

Genotyping/Scoring
Stware/program based

Figure 1. Comparison between gel-based (A) and non-gel based (B) genotyping assays

\section{KASP Assay Conversion Rate}

Generally, SNPs are transferable from one platform to another with successful conversion rates varying from 50 to $97 \%$ (Hyten et al., 2010). Successful SNP conversion rate is based on assay design success rate and conversion to working assays. LGC genomics, reports that KASP achieves $98-100 \%$ assay design success rates and $93-94 \%$ conversion to successful working assays. SNP discovery and validaton is of highly significance and it is important to validate the developed SNPs in different ways. Rasheed and coworkers (Rasheed et al., 2016) recently used this platform to convert conventional PCR markers for functional genes in wheat with more than $95 \%$ assays conversion success and it reported to be 45 times higher in throughput and $30-45 \%$ cheaper as 
compared to other available traditional methods. Zhao and coworkers (Zhao et al., 2017) also reported the successful conversion of conventional CAPS marker into KASP assay to detect AhFAD2 mutation on peanut. Successful conversion of sequence-tagged site (STS) gel based marker for $\operatorname{Lr} 21$ into KASP assay has also been reported with high throughput, accurate, inexpensive and less laborious (Neelam et al., 2013). In prairie cordgrass (Spartine pectinata) validation of DNA polymorphism has also been documented by using KASP assay. Among 121 SNPs, the assay success rate was $78.5 \%$ with 26 assays failing development (Graves et al., 2016). From pigeonpea, successful conversion of 1616 (88.4\%) out of 1827 SNPs into KASP assays has also been documented (Saxena et al., 2012). James Cockram and coworkers (Cockram et al., 2012) also reported 83\% success conversion rate. KASP genotyping system used by Kusza and co-workers on 48 polymorphisms at different caprine loci and French Alpine and Saanen goat breeds found $94.81 \%$ assay success rate and found a high locus specific and accurate genotyping solution (Kusza et al., 2018).

\section{KASP Requirements}

Quality and quantity of DNA are the two major factors determining the KASP assay success. DNA extracted from leaf and/or seed can be used. The amount of DNA required differs according to genome size; more amount of DNA is needed for species having large genome size to ensure enough copies of the genome exist at the initiation of KASP. Concentration of the extracted DNA should be taken into account on $0.8-1.0 \%$ agarose gel electrophoresis and/or spectrophotometer. For every SNP assayed, KASP needs a 5-10 ng template DNA. Purity of DNA is of the most importance as the existence of contaminations in DNA can hinder PCR. Polyphenols and polysaccharides are most common contaminants which can affect PCR. Such contaminants can be removed by adding 10-phenanthroline, polyvinylpyrrolidone-40 (PVP-40), dierhlydithiocarbamic acid, sarcosyl and sorbitol (Zhang \& Stewart, 2000). On the basis of research objectives, DNA can be extracted from one plant or bulk of different plants. For MAS in marker assisted re-current selection, DNA extracted from one plant per entry is preferable. As genotyping of each and every individual plant per accession is expensive and laborious, several reports have used bulked method (Reif et al., 2005, Warburton et al., 2010). For single cross hybrid, inbred lines, and bi-parental mapping populations, bulk method or DNA pooling (10-15 plants per sample prior to DNA extraction) is recommended for KASP genotyping. Generally, KASP works well for both methods, but genotype plots are often not sharper for bulk method than single plants. However, KASP does not work for open pollinated crops as it is not a quantitative assay. To address this issue, DNA should either be extracted from single plant or a minimum of 3-4 repeats are recommended of DNA bulk (Semagn et al., 2013).

Table 1. Minimum DNA quantity required for each sample for specific specie

\begin{tabular}{llll}
\hline Genome size & Mass (Dry DNA) & Volume and concentration (Wet DNA) & Species \\
\hline $100-750 \mathrm{Mbp}$ & $30 \mathrm{ng}$ & $6 \mu \mathrm{L}$ at $5 \mathrm{ng} / \mu \mathrm{L}$ & Rice, fruit fly, arabidopsis \\
$751-2000 \mathrm{Mbp}$ & $30 \mathrm{ng}$ & $6 \mu \mathrm{L}$ at $5 \mathrm{ng} / \mu \mathrm{L}$ & Potato, Soybean, Zebra fish \\
$2001-3500 \mathrm{Mbp}$ & $30 \mathrm{ng}$ & $6 \mu \mathrm{L}$ at $5 \mathrm{ng} / \mu \mathrm{L}$ & Human, maize, sunflower, house mouse \\
$3501-5000 \mathrm{Mbp}$ & $48 \mathrm{ng}$ & $6 \mu \mathrm{L}$ at $11 \mathrm{ng} / \mu \mathrm{L}$ & Lentil \\
$5002-10000 \mathrm{Mbp}$ & $99 \mathrm{ng}$ & $6 \mu \mathrm{L}$ at $22 \mathrm{ng} / \mu \mathrm{L}$ & Barley \\
$10001-15000 \mathrm{Mbp}$ & $150 \mathrm{ng}$ & $6 \mu \mathrm{L}$ at $33 \mathrm{ng} / \mu \mathrm{L}$ & Faba bean \\
$15001-20000 \mathrm{Mbp}$ & $198 \mathrm{ng}$ & $6 \mu \mathrm{L}$ at $44 \mathrm{ng} / \mu \mathrm{L}$ & Onion \\
$\sim 17000 \mathrm{Mbp}$ & $265 \mathrm{ng}$ & $2-3 \mu \mathrm{L}$ at $50 \mathrm{ng} / \mu \mathrm{L}$ & Wheat \\
$20001-30000 \mathrm{Mbp}$ & $300 \mathrm{ng}$ & $6 \mu \mathrm{L}$ at $67 \mathrm{ng} / \mu \mathrm{L}$ & Pine \\
$30001-50000 \mathrm{Mbp}$ & $498 \mathrm{ng}$ & $6 \mu \mathrm{L}$ at $111 \mathrm{ng} / \mu \mathrm{L}$ & Madonna lily \\
\hline
\end{tabular}

Source: http://www.lgcgroup.com

\section{KASP Primer Desiging}

Usually KASP requires three primers. Two primers having one of each fluorophore (FAM or HEX) attached as a tail and common primer. Figure (2-5) shows the possibilities to design KASP primer sets for SNP and InDel.

\section{Sampling Assayed}

DNA samples can be assayed in any 96, 384 or 1536-well micro-titter PCR plate. For 96-well plates, 5-6 $\mu \mathrm{L}$ of DNA is recommended while 2-2.5 $\mu \mathrm{L}$ of DNA is recommended for 384- and 1536-well plates. The genotyping must be carried out on at least 24 samples and two non-template control (NTC) to guarantee proper and clear clustering. 


\section{Sampling Assayed}

A minimum of two NTCs should be included on each genotyping plate. A difference in fluorescent signal intensity between the absence and presence of template DNA ensures the validity of genotyping results. Positive control (DNA samples of known genotype) should be used to eradicate false positive results. In case of assay with low allele frequency, positive control usage becomes more necessary.

KASP primer designing for 1 SNP with two forward primers and one common reverse primer

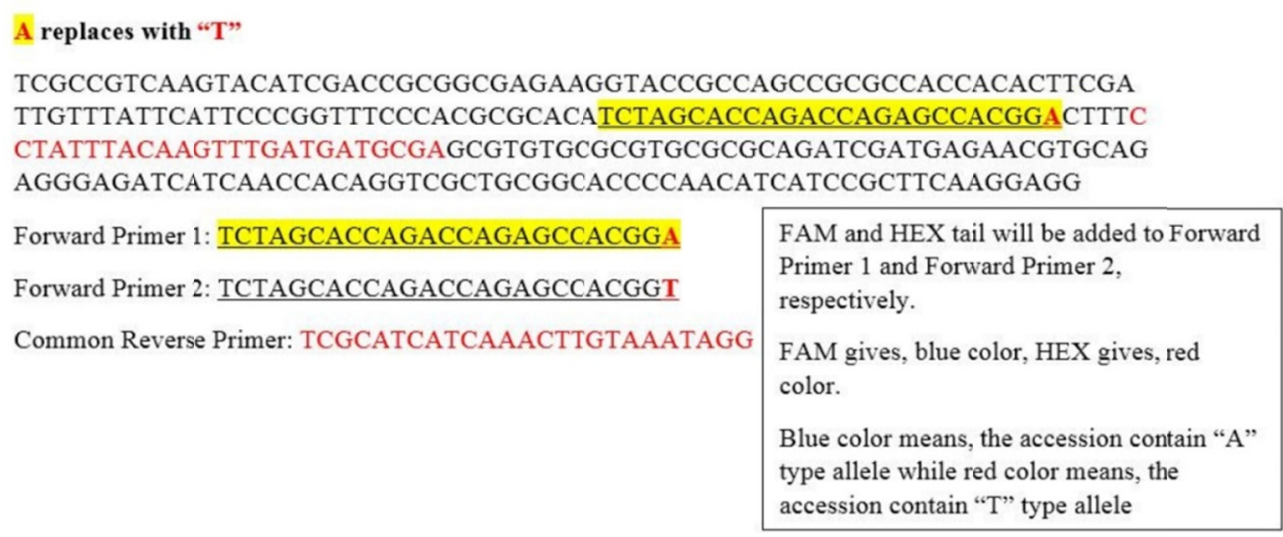

Figure 2. A hypothetical nucleotide sequnence used for KASP primer designing for $1 \mathrm{bp} \mathrm{SNP}$ with two forward primers and one common reverse primer

KASP primer designing for 1 SNP with two reverse primers and one common forward primer

C replaces with "A"

CAGGTATGCCGCCAGCCGTTCAGCTTAGCAATTCCACAAGTTGCAGTTATAGCCAAACATCT AAATACCGAGCTCAACAAAAACAGTTTTTATTAGGTGTTCCGTACTCGATTCCGGACTACAT TCACATACCAATGGACTGCCGAAACCTTCTCTCAAGGATCTTTGTTGCCAACCCAGCTACGG TGAGTACTCACT

Reverse Primer 1: TGAATGTAGTCCGGAATCGAGTACG

Reverse Primer 2: TGAATGTAGTCCGGAATCGAGTACT

Common Forward Primer: CCGAGCTCAACAAAAACAGTTTT

CGTACT (Reverse compliment for this)
Because we have sequence polymorphism
"C/A", so for second reverse primer we
will replace AGTACG to AGTACT

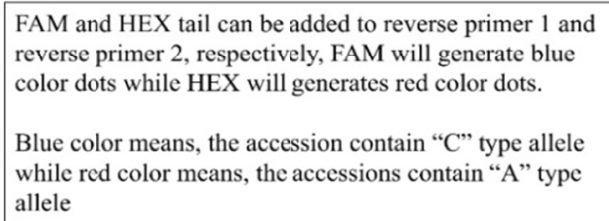

Figure 3. A hypothetical nucleotide sequnence used for KASP primer designing for $1 \mathrm{bp} \mathrm{SNP} \mathrm{with} \mathrm{two} \mathrm{reverse}$ primers and one common forward primer 
Accession 1:

KASP primer designing for 1 bp InDel

AGAGAGCTCTACGTGTACGTACGTAGCATAATGCATGCATGGAAATTCCTGGCTTTTTAAAA

CCCTGGAATTT GT GTACCTGGCATTGGAGGGTTTATGAAACGCGATTTGCATTCGGCCGGCATT

TCCCCTTAAACCTATCACTACATA

Accession 2:

AGAGAGCTCTACGTGTACGTACGTAGCATAATGCATGCATGGAAATTCCTGGCTTTTTAAAA

CCCTGGAATTTGTGTACCTGGCATTGGAGGGTTTA-

GAAACGCGATTTGCATTCGGCCGGCATTTCCCCTTAAACCTATCACTACATA

\section{Primer set 1}

(1)SNP1_AL1 GTACCTGGCATTGGAGGGTTTAT

(1)SNP1_AL1 GTACCTGGCATTGGAGGGTTTA-

(1)SNP1_T ATGCCGGCCGAATGCAAATCGCGT

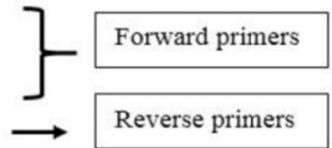

Primer set 2

(2)SNP1_AL1: GCCGGCCGAATGCAAATCGCGTTTCA

(2)SNP1_AL2: GCCGGCCGAATGCAAATCGCGTTTC

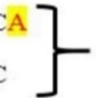

Reverse primers

(2)SNP1_T

CTGGAATTTGTGTACCTGGCATTGG

Forward primers

Figure 4. A hypothetical nucleotide sequnence used for KASP primer designing for $1 \mathrm{bp} \mathrm{InDel} \mathrm{with} \mathrm{both}$ possibilities to design primers

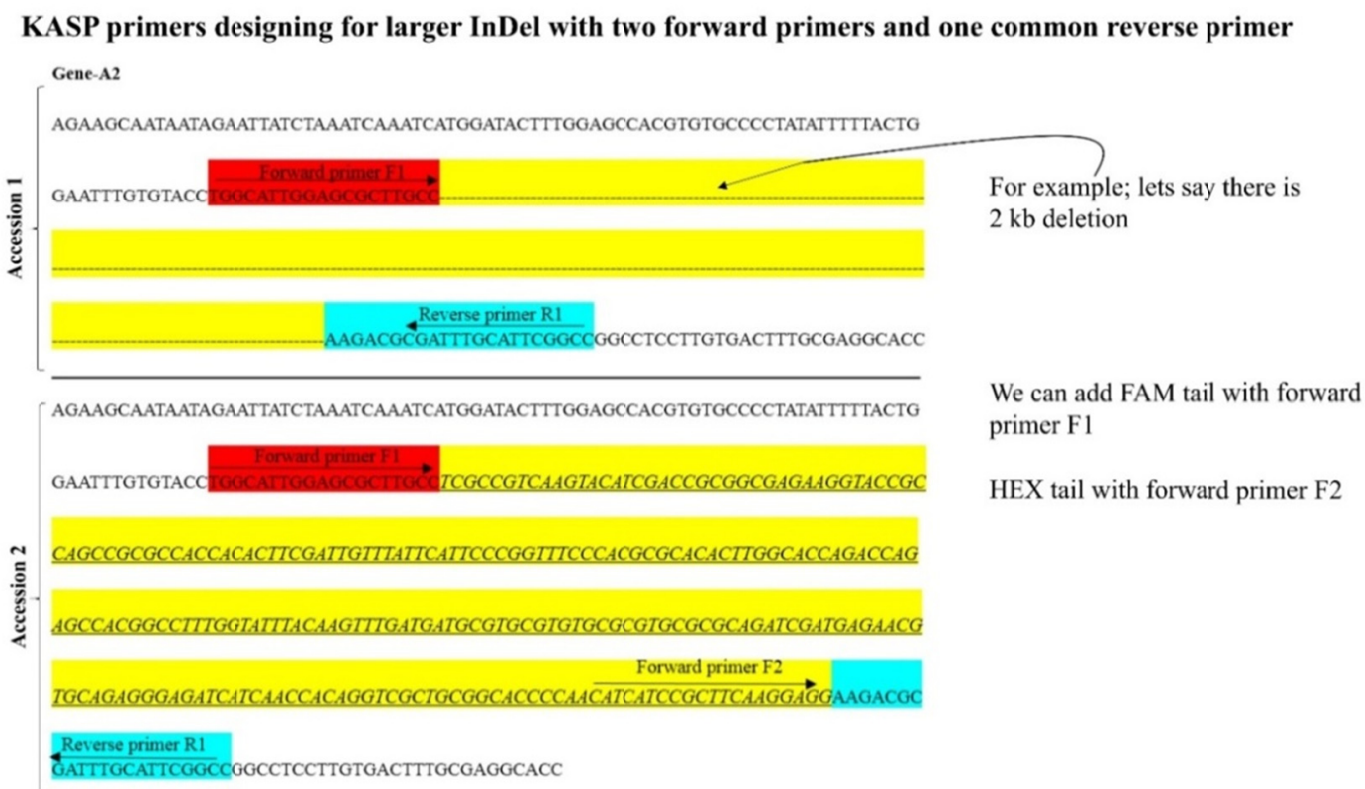

Figure 5. A hypothetical nucleotide sequnence used for KASP primer designing for larger InDel

\section{Negative and Positive Control}

A minimum of two NTCs should be included on each genotyping plate. A difference in fluorescent signal intensity between the absence and presence of template DNA ensures the validity of genotyping results. Positive control (DNA samples of known genotype) should be used to eradicate false positive results. In case of assay with low allele frequency, positive control usage becomes more necessary.

\section{Drying DNA}

DNA samples can be dried into the wells of micro-titer plate whereupon the reaction mixture must be diluted to $1 \times$ concentration (rather than $2 \times$ for liquid samples) to compensate for the absence of liquid in the well. Oven drying the DNA samples in micro-titer plates is a handy approach when doing large scale genotyping, as it allows many plates of DNA samples to be prepared in advance without the concern of sample evaporation which might otherwise change the final reagent concentrations. Dried DNA samples remain stable at room temperature 
for at least three months, if properly sealed. After arraying, the micro-titer plate having DNA samples should be centrifuged at $4000 \mathrm{rpm}$ for at least $30 \mathrm{sec}$ followed by placing the plate in drying oven at $45-60{ }^{\circ} \mathrm{C}$ for $1-2 \mathrm{~h}$ to ensure uniform dryness. Sealing the plate and storing at $4{ }^{\circ} \mathrm{C}$ (short period of time) or $-20{ }^{\circ} \mathrm{C}$ (longer period of time) is advisable if genotyping is not to be carried out straight away.

\section{Thermal Cycling Conditions}

KASP chemistry can be assayed with any standard thermal cycler. In most publications, PCR conditions are as: hot start at $95^{\circ} \mathrm{C}$ for $15 \mathrm{~min}$, followed by 10 touch down cycles $\left(95^{\circ} \mathrm{C}\right.$ for $20 \mathrm{~s}$; touch down at $65-57^{\circ} \mathrm{C}$ initially and decreasing by $0.8-1{ }^{\circ} \mathrm{C}$ per cycle for $25 \mathrm{~s}$ ), followed by $26-30$ more cycles of annealing $\left(95^{\circ} \mathrm{C}\right.$ for $10-20 \mathrm{~s}$; $57^{\circ} \mathrm{C}$ for $60 \mathrm{~s}$ ). An extension step is not necessary as the fragment length is normally less than $120 \mathrm{bp}$ (Graves et al., 2016, Rasheed et al., 2016, Zhao et al., 2017, Kusza et al., 2018).

\section{Graphical Viewing of Genotyping Data}

KASP uses fluorophores FAM and HEX for distinguishing genotypes

\begin{tabular}{|l|l|l|}
\hline Fluorophore & Excitation $(\mathrm{nm})$ & Emission $(\mathrm{nm})$ \\
\hline FAM & 485 & 520 \\
\hline HEX & 535 & 556 \\
\hline
\end{tabular}

Several data analysis software packages are available such as KlusterKaller ${ }^{\mathrm{TM}}$, Karken $^{\mathrm{TM}}$, and SNPviewer ${ }^{\mathrm{TM}}$. In these software, the FAM and HEX data can be plotted on $x$ - and $y$ - axes, respectively. Simultaneous signaling of FAM and HEX will clog the green dots at the central point between the two axis, suggesting heterzoygosity (Figure 6). Sometines, Pink color dots can also be clogged in the plot area, suggesting missing/error DNA sample. Genotypes can be determined according to sample clusters as shown in figure 6. If there are too many genotyping clusters, it is probably that a non-target polymorphism may exist in the close vicinity of the targeted SNP, hence it is impossible to detect the homozygotes and heterozygotes for the different clusters. To avoid this, at least one of the primers has to be relocated and redesigned.

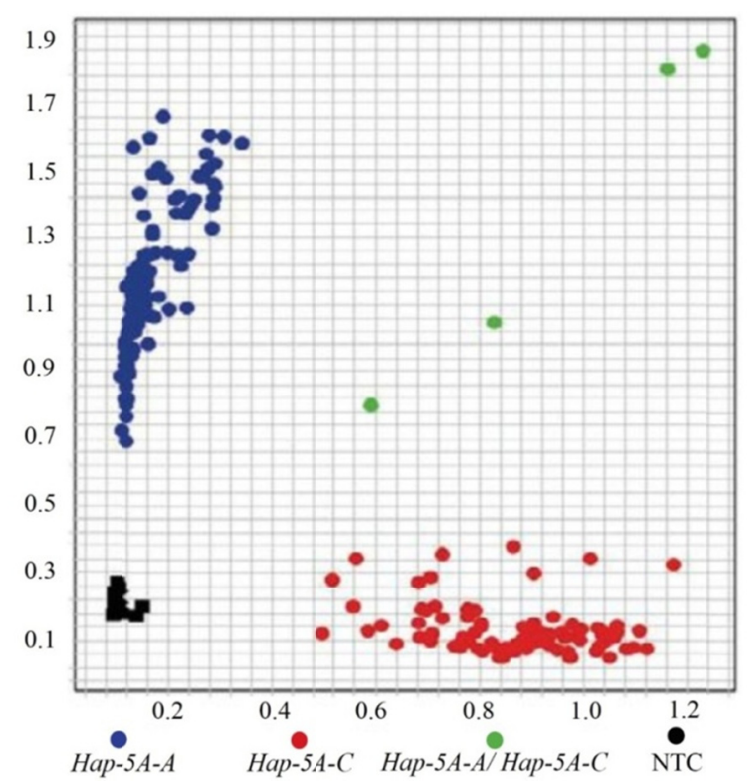

Figure 6. Allelic discrimination plot of $\operatorname{TaDRO}$, a gene associated with wheat root architectures (Zhang et al., 2018)

Note. Hap $=$ haplotype. NTC $=$ non-template control. 


\section{KASP Application}

KASP method offers more flexibility than other multiplex methods in that it can be used either for many SNPs in few sample or vice versa (Semagn et al., 2013). KASP can be used for genotyping a wide range of species for several purposes. This genotyping technology has more than 2000 published references in applications including pharmaceuticals, disease and agriculture research fields. Study conducted by Rasheed et al. (2016), developed KASP assays for various economically important traits in wheat. Some of the reported KASP assay for agronomic traits, end-use-qulaity and pre-harvesting sprouting resistance are as follows, $P p d-B 1, P p d-D 1$, VRN-A1, VRN-B1, VRN-D1, Rht-B1, Rht-D1, TaCwi-5D, TaGS-D1, TaTGW6-3A, TaGASR-A1, TaSus2-2B, TaCKX-D1, TaMoc1-7A, TaDreb-B1, Glu-A1, Glu-B1, Glu-D1, Pina-D1, Pinb-D1, Pinb-B2, Ppo-A1, Ppo-D1, Psy-A1, Psy-B1, Psy-D1, Zds-A1, TaPHS1, TaSdr-B1, TaVp-1B, TaMFT-A1. International maize and wheat improvement center (CIMMYT) has been using KASP platform for the systematic allele mining of large set of germplasm for particular functional polymorphism and QTL mapping studies. Allele mining is a favorable approach to dissect naturally occurring allelic variants at candidate genes govering important agronomic traits. A systematic allele mining of large germplasm collections for specific functional polymorphism is going on at CIMMYT courtesy KASP. QTL mapping includes identification of subset of markers that are significantly linked with one or more QTL, influencing the expression of the targeted traits. In maize, KASP assays for subset of SNPs around QTL has been developed for genotyping various fine mapping populations at CIMMYT. To validate minor QTL, marker assisted recurrent selection is a promising approach for accumulating desirable alleles from QTLs for traits governed by many genes. Plants having long juvenile phase, such as apple, which is a large woody perennial plant, are more ill-suited for traditional analysis that rely on high thoughput phenotypic selection for complex traits. Therefore, MAS gives an effective, substitute approach for pre-selecting favorable individuals for breeding tasks. SNP based markers are potential candidates for MAS. Seven SNPs near to QTLs for concentrations of soluble sugars and acids in apple have indicated markers for MAS that can assist in breeding for fruit quality (Ma et al., 2016). Haibo et al. (2018), constructed high-density genetic linkage map with 10,172 markers of apple, using SNP markers obtained through resitriction site-associated DNA sequencing (RADseq) and a segregating population of 350 seeldings from the cross of Honeycrisp and Qinguan. Thirty-three QTLs for carbon isotope composition in apple under both water stress and well-irrigated conditions were identified. Among the reported QTLs, three QTLs were stable for over 2 years under water stress on linkage groups LG8, LG15, and LG16, as validated by KASP assays. KASP assay based SNP marker, based on mapping of QTLs for $\delta^{13} \mathrm{C}$. They further stated that, genotyping through this method produced similar results to those achieved with RADseq-based SNPs. Seed Biotechnology Center of the University of California, Davis, USA applied KASP genotyping technology on commercial basis to identify and selectively breed for pepper strains with SNPs linked with resistance to Phytophthora capsici, a root rot causing factor.

High throughout and quick turnaround times of KASP genotyping technology empowered the group to validate and publish their research work ahead of the field. This technology has also been used to investigate commercial egg laying chickens for polymorphisms in exons 2-6 of the gene encoding the eggshell protein ovocalyxin-32 (OCX32) which is associated to eggshell characteristics including firmness and thickness. This method provided quick detection of a larger number of SNPs not formerly reported. In a nut shell this single-plex genotyping technology can be applied to all genotypes after validation. KASP assay was recently used to screen SNPs in various neurological disorders like Alzheimer's disease (rs75932628 of TREM2) (Benitez et al., 2014), rs1476679 of ZCWPW1 gene (Allen et al., 2015), and both rs7412 and rs429358 for Apolipoprotein E genotyping (Keogh et al., 2017), Parkinson disease (Landoulsi et al., 2017), amyotrophic lateral sclerosis (Fogh et al., 2014), polyglutamine diseases (Bettencourt et al., 2016) and genetic generalized epilepsy (VRK2 rs2947349 (Epilepsies ILAECoC et al., 2014).

\section{KASP vs Amplifluor and TaqMan}

The "Amplifluor" is another novel SNP genotyping system developed by Millipore, the company recently merged with Merck (http://www.merckmillipore.com). The basic principles of KASP and Amplifluor SPN genotyping are similar but the underlying chemistry and primer/probe assay has never been publicized by KBioscience Company for KASP (Khripin, 2006). In contrast to KASP, Milipore-Merck Company publicized the underlying chemistry, design and sequence of all components in Amplifluor SNP genotyping systems. A previous report confirms that KASP Master-mix can be used with self-developed Amplifluor system as well (Jatayev et al., 2017). The structure of universal probes (UPs) in KASP system, remains unrevealed. Although, two universal fluorescence resonance energy transfer (FRET) cassettes with fluorophores FAM and HEX are clearly mentioned on LGC Genomics web site (http://www.lgcgroup.com) but the information regarding type of quencher UPs is still unrevealed for KASP genotyping. At the moment, we only speculate the UPs general 
structures in both KASP and Amplifluor like system are similar, if not identical. Amplifluor like SNP genotyping system are considered 10-20 folds less expensive as compared to KASP as once-ordered stock of two UPs labelled with different dyes can used to analyze a number of SNPs. Although, Amplifluor like SNP genotyping system is considered less expensive, but the perfect optimization of UPs and associated well organized business and marketing makes KASP markers very popular amongst wider range of researchers (Jatayev et al., 2017).

KASP and TaqMan assays have common advanatgaes, i.e. less laborious, cost effective, more tolerance of variability in DNA quality and quantity. Both assays could be used for large sample numbers in much lesser time bypassing post-PCR handling (He et al., 2014). These properties are important to deal with larger scale genotyping. Beside this, KASP and TaqMan assays are appropriate to be used on any real-time PCR machine. TaqMan requires tagging of the SNP specific primers which increased the cost of assay. Therefore, KASP genotyping assay showed a cost saving option. The current KASP reaction costs five time cheaper than TaqMan. Furthermore, a study of SNP selection for soybean genotyping has stated that KASP assay cost 0.005 US\$ as compared to 0.238 US\$ for TaqMan (Yuan et al., 2014). Quite a large number of publications with reference to KASP analysis indicate a large interest of researchers in this technology.

\section{KASP Drawbacks and Alternative SNP Genotying Techniques}

Although KASP is a high throughput genotyping assay yet reports are there stating $0.7-1.6 \%$ error rate. KASP is also probably not suitable when the mutant allele is present in very low quantities. When there is less than 5\% quantity of mutant allele as compared to wild type allele, then it is difficult to differentiate between the individual/sample containing mutant or wild type DNA.

To deal with the low quantity of mutant DNA, several other SNP genotyping techniques are available. CastPCR $^{\mathrm{TM}}$ (http://www.lifetechnologies.com) is a technique which blocks the annealing and elongation steps of the allele that is not your target one (Roma et al., 2013). ddPCR ${ }^{\mathrm{TM}}$ is another technique (http://www.bio-rad.com), which enables the absolute quantitation of nucleic acids in a sample (Hindson et al., 2011).

\section{Acknowledgements}

This work was supported by grants from the National Key Research and Development Program of China (2016YFD0100302).

\section{References}

Allen, M., Kachadoorian, M., Carrasquillo, M. M., Karhade, A., Manly, L., Burgess, J. D., Nilufer, E. T. (2015). Late-onset Alzheimer disease risk variants mark brain regulatory loci. Neuorology Genetics, 1(2), e15. https://doi.org/10.1212/NXG.0000000000000012

Bettencourt, C., Hensman-Moss, D., Flower, M., Wiethoff, S., Brice, A., Goizet, C., Jones, L. (2016). DNA repair pathways underlie a common genetic mechanism modulating onset in polyglutamine diseases. Annals of Neurology, 79(6), 983-990. https://doi.org/10.1002/ana.24656

Benitez, B. A., Jin, S. C., Guerreiro, R., Graham, R., Lord, J., Harold, D., Carlos, C. (2014). Missense variant in TREML2 protects against Alzheimer's disease. Neurobiology of Aging, 35(6), 1510e1519-26. https://doi.org/10.1016/j.neurobiolaging.2013.12.010

Cockram, J., Jones, H., Norris, C., \& O'Sullivan, D. M. (2012). Evaluation of diagnostic molecular markers for DUS phenotypic assessment in the cereal crop, barley (Hordeum vulgare ssp. L.). Theoretical \& Applied Genetics, 125(8), 1735-1749. https://doi.org/10.1007/s00122-012-1950-3

Epilepsies ILAECoC. (2014). Genetic determinants of common epilepsies: A meta-analysis of genome-wide association studies. The Lacent Neurology, 13(9), 893-903. https://doi.org/10.1016/S1474-4422(14)70171-1

Fogh, I., Ratti, A., Gellera, C., Lin, K., Tiloca, C., Moskvina, V., Zheng, J. G. (2013). A genome-wide association meta-analysis identifies a novel locus at $17 \mathrm{q} 11.2$ associated with sporadic amyotrophic lateral sclerosis. Human Molecular Genetics, 23(8), 2220-2231. https://doi.org/10.1093/hmg/ddt587

Graves, H., Rayburn, A. L., Gonzalez-Hernandez, J. L., Nah, G., Kim, D. S., \& Lee, D. K. (2016). Validating DNA polymorphisms using KASP assay in prairie cordgrass (Spartina pectinata Link) populations in the U.S. Frontiers in Plant Science, 6(1271). https://doi.org/10.3389/fpls.2015.01271

Haibo, W., Shuang, Z., Mao, K., Qinglong, D., Bowen, L., Chao, L., Fengwang, M. (2018). Mapping QTLs for water use efficiency reveals the potential candidate genes involved in regulating the trait in apple under drought stress. BMC Plant Biology, 18(136). 
Hindson, B. J., Ness, K. D., Masquelier, D. A., Belgrader, P., Heredia N. J., Makarewicz, A. J., Colston, B. W. (2011). High-thoughput droplet PCR system for absolute quantitation of DNA copy number. Analytical Chemistry, 83(22), 8604-8610. https://doi.org/10.1021/ac202028g

He, C., Holme, J., \& Anthony, J. (2014). SNP genotyping: The KASP assay. Methods in Molecular Biology, (Clifton, N. J.). 1145, 75-86. https://doi.org/10.1007/978-1-4939-0446-4_7

Hyten, D. L., Cannon, S. B., Song, Q., Weeks, N., Fickus, E. W., Shoemaker, R. C., Cregan, P. B. (2010). High-throughput SNP discovery through deep resequencing of a reduced representation library to anchor and orient scaffolds in the soybean whole genome sequence. BMC Genomics, 11(1), 38. https://doi.org/10.1186/1471-2164-11-38

Jatayev, S., Kurishbayev, A., Zotova, L., Khasanova, G., Serikbay, D., Zhubatkanov, A., Shavrukov, Y. (2017) Advantages of amplifluor-like SNP markers over KASP in plant genotyping. BMC Plant Biology, 17(Suppl 2), 254. https://doi.org/10.1186/s12870-017-1197-X

Keogh, M. J., Wei, W., Wilson, I., Coxhead, J., Ryan, S., Rollinson, S., Chinnery, P. F. (2017). Genome Research, 27(1), 165-73. https://doi.org/10.1101/gr.210609.116

Khripin, Y. (2006). High-throughput genotyping with energy transfer-labeled primers. Methods in Molecular Biology, (Clifton, N.J.) 335, 215-240. https://doi.org/10.1385/1-59745-069-3:215

Kumpatla, S., Buyyarapu, R., Abdurakhmonov, I., \& Mammadov, J. (2012). Genomics-assisted plant breeding in the 21st Century: Technological Advances and Progress. In I. Y. Abdurakhmonov (Ed.), Plant Breeding. IntechOpen. https://doi.org/10.5772/37458

Kusza, S., Cziszter, L. T., Ilie, D. E., Sauer, M., Padeanu, I., \& Gavojdian, D. (2018). Kompetitive Allele Specific PCR (KASP ${ }^{\text {TM}}$ ) genotyping of 48 polymorphisms at different caprine loci in French Alpine and Saanen goat breeds and their association with milk composition. PeerJ, 6 , e4416. https://doi.org/10.7717/peerj.4416

Landoulsi, Z., Benromdhan, S., Djebara, M. B., Damak, M., Dallali, H., Kefi, R., \& Gouider, R. (2017). Using KASP technique to screen LRRK2 G2019S mutation in a large Tunisian cohort. BMC Medical Genetics, 18(70), https://doi.org/10.1186/s12881-017-0432-5

Ma, B., Zhao, S., Wu, B., Wang, D., Peng, Q., Qwiti, A., \& Li, S. (2016). Construction of a high density linkage map and its application in the identification of QTLs for soluble sugar and organic acid components in apple. Tree Genetics \& Genomes, 12(1). https://doi.org/10.1007/s11295-015-0959-6

Mccouch, S. R., Teytelman, L., Xu, Y., Lobos, K. B., Clare, K., Walton, M., Fu, B., \& Xing, Y. (2002). Development and mapping of 2240 new SSR markers for rice (Oryza sativa L.) (Supplement). DNA Research, 9(6), 257-279. https://doi.org/10.1093/dnares/9.6.257

Neelam, K., Brown-Guedira, G., \& Huang, L. (2013). Development and validation of a breeder-friendly KASPar marker for wheat leaf rust resistance locus Lr21. Molecular Breeding, 31(1), 233-237. https://doi.org/10.100 7/s11032-012-9773-0

Rasheed, A., Hao, Y., Xia, X., Khan, A., Xu, Y., Varshney, R. K., \& He, Z., (2017). Crop breeding chips and genotyping platforms: progress, challenges, and perspectives. Molecular Plants, 10(8), 1047-1064. https://doi.org/10.1016/j.molp.2017.06.008

Rasheed, A., Wen, W., Gao, F., Zhai, S., Jin, H., Liu, J., \& He, Z. (2016). Development and validation of KASP assays for genes underpinning key economic traits in bread wheat. Theoratical \& Applied Genetics, 129(10), 1843-1860. https://doi.org/ 10.1007/s00122-016-2743-X

Reif, J. C., Hamrit, S., Heckenberger, M., Schipprack, W., Peter Maurer, H., Bohn, M., \& Melchinger, A. E. (2005). Genetic structure and diversity of European flint maize populations determined with SSR analyses of individuals and bulks. Theoretical \& Applied Genetics, 111(5), 906-913. https://doi.org/10.1007/s0012 2-005-0016-1

Roma, C., Esposito, C., Rachiglio, A. M., Pasquale, R., Iannaccone, A., Chiccchinelli, N., \& Normanno, N. (2013). Detection of EGFR mutation by TaqMan mutation detection assays powered by competitive allele specific TaqMan PCR technology. BioMed Research Internation, 9. https://doi.org/10.1155/2013/385087

Saxena, R. K., Penmetsa, R. V., Upadhyaya, H. D., Kumar, A., Carrasquilla-Garcia, N., Schlueter, J. A., Varshney, R. K. (2012). Large-scale development of cost-effective single-nucleotide polymorphism marker assays for 
genetic mapping in pigeonpea and comparative mapping in legumes. DNA Research, 19(6), 449-461. https://doi.org/10.1093/dnares/dss025

Schlotterer, C. (2004). The evolution of molecular marker - just a matter of fashion? Nature Reviews Genetics, 5(1), 63. https://doi.org/10.1038/nrg1249

Semagn, K., Babu, R., Hearne, S., \& Olsen, M. (2013). Single nucleotide polymorphism genotyping using Kompetitive Allele Specific PCR (KASP): Overview of the technology and its application in crop improvement. Molecular Breeding, 33(1), 1-14. https://doi.org/10.1007/s11032-013-9917-x

Smith, J. S. C., Chin, E. C. L., Shu, H., Smith, O. S., Wall, S. J., Senior, M. L., Ziegle, J. (1997). An evaluation of the utility of SSR loci as molecular markers in maize (Zea mays L.): comparisons with data from RFLPs and pedigree. Theoretical \& Applied Genetics, 95(1-2), 163-173. https://doi.org/10.1007/s001220050544

Somers, D. J., Isaac, P., \& Edwards, K. (2004). A high-density microsatellite consensus map for bread wheat (Triticum aestivum L.). Theoretical \& Applied Genetics, 109(6), 1105-1114. https://doi.org/10.1007/s00122-004-1740-7

Varshney, R., Marcel, T., Ramsay, L., Russell, J., Roder, M., Stein, N., \& Graner, A. (2007). A high density barley microsatellite consensus map with 775 SSR loci. Theoretical \& Applied Genetics, 114(6), 1091-1103. https://doi.org/10.1007/s00122-007-0503-7

Varshney, R. K., Graner, A., \& Sorrells, M. E. (2005). Genic microsatellite markers in plants: features and applications. Trends in Biotechnologyi, 23(1), 48-55. https://doi.org/10.1016/j.tibtech.2004.11.005

Warburton, M., Setimela, P., Franco, J., Cordova, H., Pixley, K., Bänziger, M., \& MacRobert, J. (2010). Toward a cost-effective fingerprinting methodology to distinguish maize open-pollinated varieties. Crop Science, 50(2), 467-477. https://doi.org/10.2135/cropsci2009.02.0089

$\mathrm{Xu}, \mathrm{Y}$., \& Crouch, J. H. (2008). Marker-assisted selection in plant breeding: From publications to practice. Crop Science, 48(2), 391-407. https://doi.org/10.2135/cropsci2007.04.0191

Yuan, J., Wen, Z., Gu, C., \& Wang, D. (2014). Introduction of high throughput and cost effective SNP genotyping platforms in soybean. Plant Genetics Genomics and Biotechnology, 1(3), 1125-1131. https://doi.org/10.5147/pggb.v2i1.155

Zhang, J., \& Stewart, J. M. (2000). Economical and rapid method for extracting cotton genomic DNA. Journal of Cotton Science, 4(3), 193-201.

Zhang, W. J., Li, T., Qin, L., Zhao, J., Zhao, J. J., Liu, H., Zhang, X. Y. (2018). TaDRO, A Gene Associated with Wheat Root Architectures, Its Global Distribution and Evolution in Breeding. Scientia Agricultura Sinica, 51(10), 1815-1829.

Zhao, S., Li, A., Li, C., Xia, H., Zhao, C., Zhang, Y., Wang, X. (2017). Development and application of KASP marker for high throughput detection of AhFAD2 mutation in peanut. Electronic Journal of Biotechnology, 25, 9-12. https://doi.org/10.1016/j.ejbt.2016.10.010

\section{Copyrights}

Copyright for this article is retained by the author(s), with first publication rights granted to the journal.

This is an open-access article distributed under the terms and conditions of the Creative Commons Attribution license (http://creativecommons.org/licenses/by/4.0/). 